\title{
Visual disturbance in Diabetes Mellitus; don't be blind to alternatives to retinopathy
}

Smith TD, ${ }^{\dagger}$ Mukherjee A, ${ }^{\dagger}$ Dr S Ehtisham,$\ddagger$ Tomlinson ND ${ }^{\dagger}$

$\dagger$ Royal Oldham Hospital, Pennine Acute Hospitals NHS Trust $\ddagger$ Central Manchester University Hospitals NHS Foundation Trust

\section{Case Summary}

- Diabetes Mellitus diagnosed at 4 years old. Vision problems began at age 8

- No retinopathy. Impaired colour vision and significant bilateral optic disc pallor

- Normal neurological examination and cranial MRI

- No mitochondrial DNA deletion to suggest Leber's optic atrophy

- Parental consanguinity suggests a possible genetic aetiology

- Child is homozygous for WFS1 gene mutation (type 1 Wolfram Syndrome)

- Use of an insulin pump provides additional benefits given her vision problems

- Diabetes control had been poor, which hindered surveillance for the onset of Diabetes Insipidus

- Developed Diabetes Insipidus at 13 years old

- Hearing currently unaffected and renal ultrasound normal

- In view of the family history, when their 2 year old sibling developed Diabetes Mellitus aged 2 they were tested for the WFS1 mutation, which was present.

Wolfram Syndrome was diagnosed before the onset of optic atrophy in this child

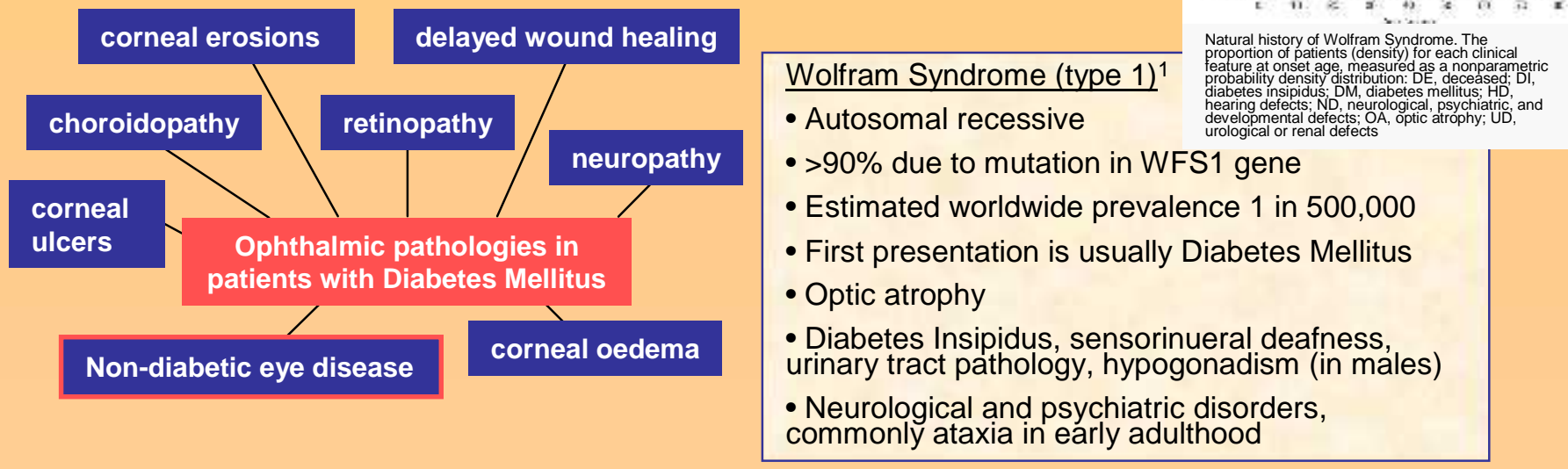

\section{Learning points}

- Ophthalmic pathologies are well-recognised complications of Diabetes Mellitus

- Retinopathy screening is indicated at all ages ${ }^{2}$

- Alternative ophthalmic/non-ophthalmic aetiologies must be considered

- Diabetes Mellitus with optic atrophy should be investigated for Wolfram Syndrome

- Additional diagnoses cause cumulative stresses on the child and family, which can hinder disease management and treatment compliance

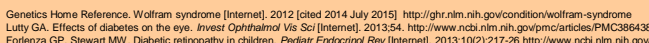

